\title{
Vibration Characteristic Analysis of the Cross-type Joint and Rzeppa Joint of the SS400 Transmission Shaft on Micro-vehicle
}

\author{
HUANG Feng-yun ${ }^{1, a}$, WEI Ding ${ }^{1, b^{*}}$, SHI Lei i, c $^{2}$
}

${ }^{1}$ School of Mechanical and Electronic Engineering, Wuhan University of Technology, Wuhan, China

${ }^{2}$ SAIC MOTOR Commercial Vehicle Technology Center, Shanghai, China

abigwasp@163.com, bweiding0529@163.com, '296174878@qq.com

Keywords: Rzeppa joint, SS400 transmission shaft, Vibration characteristic, ADAMS.

\begin{abstract}
Taking a micro-vehicle SS400 transmission shaft as the research object, the dynamic characteristics of the cross-type universal joint and the rzeppa universal joint were analyzed. And it explains that the rzeppa universal joint can reduce the transmission shaft vibration by improving the stress performance of the shaft tube support. The ADAMS virtual prototype model of the cross-type transmission shaft assembly and the rzeppa transmission shaft assembly were established respectively. The main tube bending deformation and the vertical vibration acceleration of the middle support of the two kinds of transmission shaft could be obtained through simulation. The simulation results show that the vibration characteristic of the rzeppa transmission shaft is superior to the cross-type transmission shaft. It provides reference for the applicability and rationality of the rzeppa transmission shaft.
\end{abstract}

\section{Introduction}

Transmission shaft is one of the key components of automobile transmission system. It's easy to produce bending vibration, torsional vibration and their coupling vibration in high-speed rotation, thereby affecting the transmission efficiency of the transmission shaft [1]. As the input excitation of the main reduction gear, the torsional vibration of the transmission shaft will cause great fluctuations in the torque from transmission shaft to main reduction gear, increase the collision of the main reducer gear when meshed, and intensify vibration and noise of the main reducer gear. In order to achieve the purpose of vibration and noise reduction, the applicability and rationality of the rzeppa transmission shaft assembly are studied by combining theory and simulation analysis.

\section{Dynamics Analysis of the Universal Joint}

Universal joint includes rzeppa type and cross type. Rzeppa universal joint and cross-type universal joint are essentially different in dynamics. Compared with cross-type universal joint, rzeppa universal joint has the advantages of compact structure, small vibration and high transmission efficiency. Since rzeppa universal joint has the characteristic of constant velocity, it is used in the transmission shaft to reduce and avoid the vibration and noise and other defects caused by the none constant velocity characteristic of the cross-type universal joint.

Force Analysis of Cross-Type Universal Joint. Structure diagram of single cross-type universal joint is shown in Fig. 1:

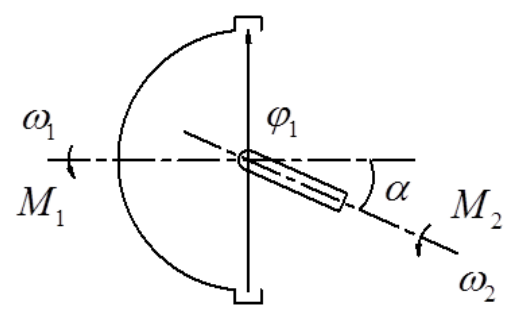

Fig. 1 Structure diagram of cross-type universal joint. 
For a single cross shaft universal joint, assuming that the internal friction of universal joint and its deformation were ignored, that is to say universal joint has no energy loss in transmission process. When the input shaft and output shaft of the universal joint form a certain angle $\alpha$ and the rotation angle of the input shaft is $\varphi_{1}$, the angular velocity of the input shaft $\omega_{1}$, the input torque $M_{1}$ and the angular velocity of the output shaft $\omega_{2}$, the output torque $M_{2}$ form the following relations [2]:

$$
\frac{M_{1}}{M_{2}}=\frac{\omega_{2}}{\omega_{1}}=\frac{\cos \alpha}{1-\sin ^{2} \alpha \cos ^{2} \varphi_{1}}
$$

At the same time, when the input shaft and output shaft of the universal joint form a certain angle $\alpha$, the input torque and output torque vector will be at an angle to each other. Since the two torques are not in one direction, the universal joint can't reach balance in the two torques. Therefore, in the cross-type universal joint there must be another additional torque, called additional bending moment. The additional bending moment of the cross-type universal joint is related to the universal joint angle $\alpha$, the rotation angle of the input shaft $\varphi_{1}$ and the rotation angle of the output shaft $\varphi_{2}$. The additional moment of the input shaft $T_{1}$ and the additional moment of the output shaft $T_{2}$ have the following relations [3]:

$$
T_{1}=-M_{1} \cos \varphi_{1} \tan \alpha, T_{2}=\frac{\sin ^{2} \varphi_{1} \sin \alpha}{\sin \varphi_{2}} M_{1}
$$

The above equations show that the value of the additional bending moment varies with the angle of the universal joint when the rotation angle of the input shaft is fixed. This additional moment makes the main tube of the transmission shaft bending, and causes the transmission shaft coupling vibrations of bending and torsional generated in high-speed rotation, and reduces the life of the transmission shaft.

The counterforce at the shaft tube support location of input shaft and output shaft are $F_{1}$ and $F_{2}$, and the distance between the center of the universal joint and the support is $L$. When the rotation angle of the input shaft $\varphi_{1}=0$, Eq. (2) shows that, the input shaft additional bending moment $T_{1}=M_{1} \tan \alpha$, the output shaft additional bending moment $T_{2}=0$. According to the force balance of the universal joint, the counterforce at the shaft tube support location of input shaft and output shaft are:

$$
F_{1}=\frac{M_{1} \tan \alpha}{L}, F_{2}=0
$$

When the rotation angle of the input shaft $\varphi_{1}=\pi / 2$, also by Eq. (2) shows that, the input shaft additional bending moment $T_{1}=0$, the output shaft additional bending moment $T_{2}=M_{1} \sin \alpha$. The counterforce at the shaft tube support location of input shaft and output shaft are:

$$
F_{1}=0, F_{2}=\frac{M_{1} \sin \alpha}{L}
$$

Study and analysis of Eq. (3) and (4) shows that, to use the cross-type universal joint in transmission shaft will generate additional moment when the input shaft and output shaft of the universal joint form a certain angle. The additional bending moment on the one hand will cause the transmission shaft tube bending deformation. On the other hand, it will generate dynamic counterforce at the shaft tube support location. Both of them as an excitation will cause the car drive transmission system bending vibration.

Force Analysis of Rzeppa Universal Joint. Rzeppa universal joint falls into the category of constant velocity universal joint, and the input shaft angular velocity $\omega_{3}$ and the output shaft angular velocity $\omega_{4}$ is equal, regardless of the shaft angle $\beta$. Assuming that there was no energy loss in transmission process, the output torque $M_{4}$ would be equal to the input torque $M_{3}[4,5]$ :

$$
\omega_{3}=\omega_{4}, M_{3}=M_{4}
$$

When the input shaft and output shaft of the rzeppa universal joint form a certain angle $\beta$, as well as cross-type universal joint, since the input torque and output torque are not in one direction, there must be an additional torque in the rzeppa universal joint. Set $V_{1}, T_{3}$ and $V_{2}, T_{4}$ respectively 
represent the bending moment components in two directions at the input shaft and output shaft section $S_{1}$ and $S_{2}$, as shown in Fig. 2:

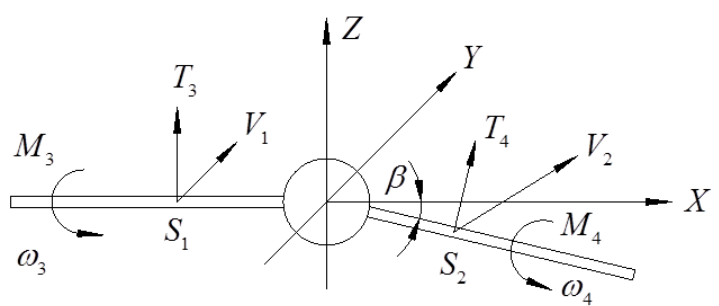

Fig. 2 Structure diagram of rzeppa universal joint.

Due to the external force on the input shaft and output shaft of the universal joint only torque, the various torque on the section $S_{1}$ and $S_{2}$ can be obtained according to the equilibrium condition of the universal joint:

$$
\begin{aligned}
& M_{4} \cdot \cos \beta+T_{4} \cdot \sin \beta-M_{3}=0 \\
& V_{1}+V_{2}=0 \\
& M_{4} \cdot \sin \beta+T_{4} \cdot \cos \beta+T_{3}=0
\end{aligned}
$$

By the Eqs. (6) and (8) shows that when ignoring the internal friction of universal joint, that is, there is no energy loss in transmission process:

$$
T_{3}=T_{4}=M_{3} \tan \frac{\beta}{2}
$$

The bending moment component on another direction can be calculated by the principle of virtual displacement method: Assuming that the input shaft is fixed, the output shaft is rotated $d \beta$ angle around the $\mathrm{Y}$-axis and it rotates $d \varphi$ upon its axis, at this time the vector $T_{4}$ and $M_{4}$ are perpendicular to the vector $d \beta$, the vector $T_{4}$ and $V_{2}$ are perpendicular to the vector $d \varphi$, so the work done by torque $T_{4}$ and $M_{4}$ to the angle $d \beta$ is 0 , and the work done by torque $T_{4}$ and $V_{2}$ to the turning angle $d \varphi$ is 0 . If the energy loss of mechanical friction is not considered, the sum of virtual work equals to zero. The equation can be expressed as:

$$
-V_{2} \cdot d \beta+M_{4} \cdot d \varphi=0
$$

According to the constant velocity characteristic, when the angle $\beta$ changes, the difference of turning angle identically equals to zero, that is $d \varphi / d \beta=0$, so:

$$
V_{1}=V_{2}=0
$$

Therefore the counterforce at the shaft tube support location of input shaft and output shaft of the rzeppa universal joint are:

$$
F_{3}=F_{4}=\frac{M_{3} \tan \frac{\beta}{2}}{L}
$$

Analysis of Eqs. (9), (11) and (12) shows that, the additional moment at the input and output shafts of rzeppa universal joint is only related with the angle $\beta$, and the two torque direction are opposite and perpendicular to the input and output shafts, and act on the plane of rzeppa universal joint axes, when the input torque of universal joint $M_{3}$ is constant. So the additional moment of the rzeppa universal joint will not generate dynamic counterforce at the shaft tube support location.

Contrastive Analysis of the Cross-type Universal Joint and Rzeppa Universal Joint. When the angle of the universal joint is less than $\pi / 2$, according to (3), (4) and (12), it can be expressed as:

$$
F_{3}=F_{4}=\frac{M_{3} \tan \frac{\beta}{2}}{L}<\frac{M_{1} \sin \alpha}{L}<\frac{M_{1} \tan \alpha}{L}
$$


Analysis of Eq. (13) shows that, the counterforce at the shaft tube support location of the rzeppa universal joint is less than cross-type universal joint, and the additional moment of the rzeppa universal joint will not generate dynamic counterforce, when the transmission torque and the angle of the two universal joints are the same. So the vibration characteristic of the rzeppa universal joint is superior to cross-type universal joint.

\section{ADAMS Dynamics Simulation Analysis of the Transmission Shaft}

The transmission shaft of the researched vehicle is in the form of two section shaft tube and three universal joints, and its material is SS400. SS400 is one of the Japanese steel, its material properties could be obtained through checking tables or material test. The transmission shaft assembly model was established by UG. Import the model to ADAMS without gasket and bolt, adding constraints, and ignoring the influence of friction and collision in the universal joint, the middle support bearing and the sliding spline on vibration characteristic of transmission shaft [6]. And enter the relevant material properties of SS400 to the parameter table. As shown in Fig. 3, the virtual prototype model of the transmission shaft which middle universal joint is rzeppa type was created. And the virtual prototype model of the transmission shaft which middle universal joint is cross-type could be created similarly.

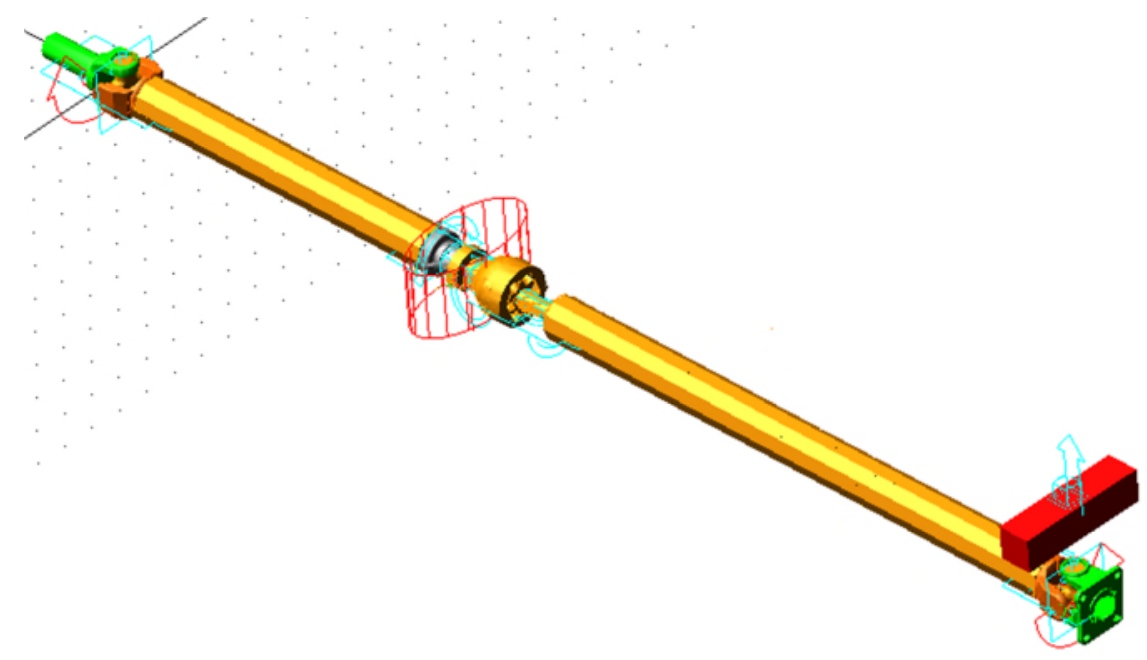

Fig. 3 The rzeppa transmission shaft.

The rotate speed and torque of the transmission shaft of the vehicle commonly used are shown in Table 1. Taking them as an initial condition of the shaft simulation, analysis was made of the two kinds of transmission shaft of the main tube bending deformation and the vertical vibration acceleration of the middle support.

Table 1. The rotate speed and torque of the transmission shaft.

\begin{tabular}{|c|c|c|c|c|c|c|c|}
\hline rotate speed $(\mathrm{r} / \mathrm{min})$ & 657.89 & 789.47 & 921.05 & 1052.6 & 1184.2 & 1250.0 & 1500.0 \\
\hline torque $(\mathrm{N} \cdot \mathrm{m})$ & 376.20 & 395.20 & 410.40 & 418.00 & 414.20 & 198.00 & 208.00 \\
\hline rotate speed $(\mathrm{r} / \mathrm{min})$ & 1750.0 & 2000.0 & 2250.0 & 2500.0 & 2857.1 & 3214.3 & \\
\hline torque $(\mathrm{N} \cdot \mathrm{m})$ & 216.00 & 220.00 & 218.00 & 151.20 & 154.00 & 152.60 & \\
\hline
\end{tabular}

Analysis of the Main Tube Bending Deformation. The main tube bending deformation of the rzeppa transmission shaft and the cross-type transmission shaft can be achieved by the simulation, as shown in Fig. 4.

Analysis of Fig. 4 shows that, compared with the cross-type transmission shaft, the main tube bending deformation of rzeppa transmission shaft is about $0.025 \mathrm{~mm}$, and the main tube bending deformation of the cross-type transmission shaft is greater than that of the rzeppa transmission shaft at various speeds, so the result obtained by comparing the main tube bending deformation of the 
two types of transmission shaft shows that the transmission shaft which uses the rzeppa universal joint can reduce the main tube bending deformation, so as to reduce the vibration of transmission shaft. Because of the cross-type transmission shaft generally with low frequency vibration, the main tube bending deformation of cross-type transmission shaft is very great when the rotate speed between 500 1100r/min and dropped down around 1200 rotate speed.

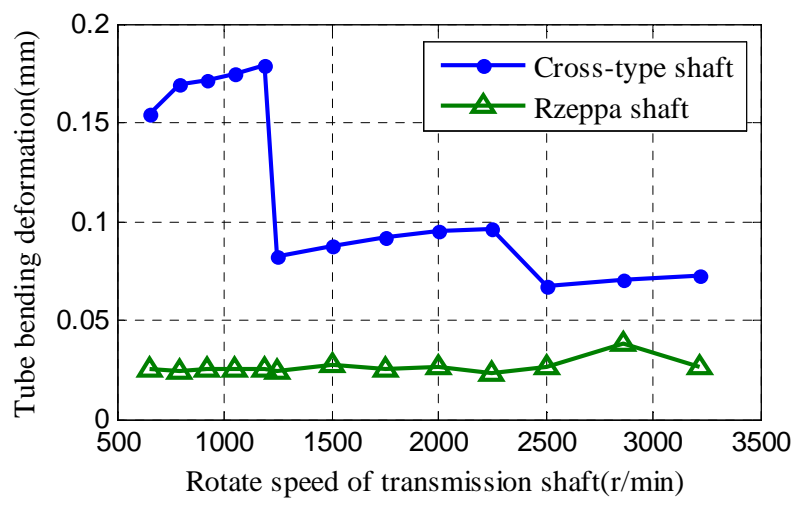

Fig. 4 The main tube bending deformation.

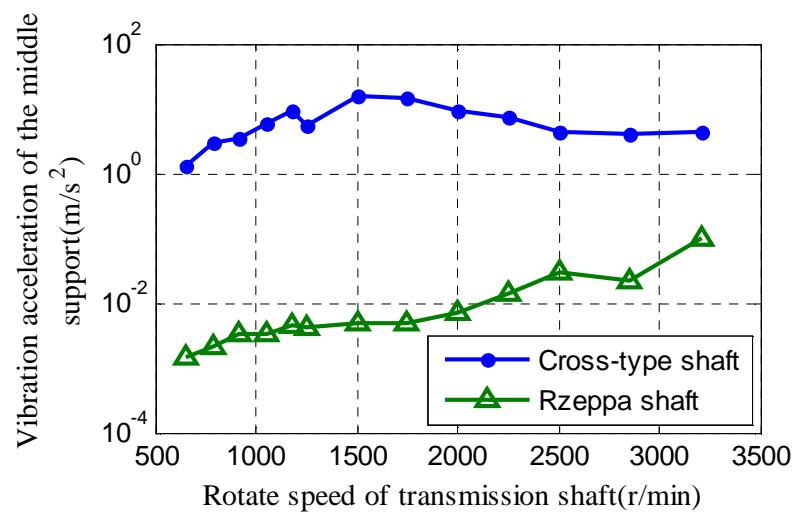

Fig. 5 The vibration of the middle support.

Analysis of the Vibration of the Middle Support. The vibration of the middle support of the transmission shaft can be transmitted to the vehicle body through the bracket of the automobile chassis. It will affect the comfort of the vehicle if the vibration of the middle support is too large. The vertical vibration accelerations of the rzeppa transmission shaft and the cross-type transmission shaft can be achieved by the simulation, as shown in Fig. 5.

Analysis of Fig. 5 shows that, the minimum value of the vertical vibration acceleration of the middle support of the cross-type transmission shaft is $1.27 \mathrm{~m} / \mathrm{s}^{2}$, and the maximum value is $15.18 \mathrm{~m} / \mathrm{s}^{2}$. And the maximum value of the vertical vibration acceleration of the middle support of the rzeppa transmission shaft is $0.1 \mathrm{~m} / \mathrm{s}^{2}$, which is far less than that of the cross-type transmission shaft. This shows that the vibration of the middle support of the transmission shaft can be reduced effectively, and the performance of the transmission shaft can be improved by using the rzeppa universal joint in the transmission shaft.

\section{Summary}

1. The dynamic counterforce is generated at the support of the cross axle type universal joint shaft tube by the analysis of dynamics characteristic of the cross-type joint and the rzeppa universal joint. And the counterforce of the rzeppa universal joint is constant and less than the universal cross-type joint.

2. The vibration characteristic of the rzeppa transmission shaft is better than the cross-type transmission shaft by the dynamics analysis of the transmission shaft in ADAMS when the material of transmission shaft is SS400.

\section{References}

[1] Z. M. Yang, Transmission Shaft and Universal Joint (China Communications Press, Beijing 1986).

[2] Q. Zhao, Y. K. Shi, Y. Sun, Study on Automobile Noise \& Vibration of Causing by Transmission Shaft with Cross- type Universal Joint, J. Mech. Transmission, 35(10) (2011) 70-76.

[3] S. X. Zhao, H. Xiao, Y. T. Liu, Research on Dynamic Analysis and Torque Testing of Cross-type Universal Joint, Metallurgical Industry Automation (2009). 
[4] R. Kazemi, B. Hamedi, M. Izadkhah, The Vibrational Improvement of the Two-Piece Driveline of the Passenger Car, SAE 2002 World Congress (2002).

[5] Z. H. Qi, G. Wang, Z. G. Zhang, Kinematic Analysis and Simulation of the Steel Balls for Rzeppa Constant Velocity Joint, J. Mech. Eng. 48(03) (2012) 147-153.

[6] H. C. Zhou, H. H. Zhai, J. Yang, Vibration Analysis of Propeller Shaft and Rear Axle Housing Systemic Based on ADAMS, J. Chongqing Jiaotong U. (Natural Science) (2015). 